Check for updates

Cite this: RSC Adv., 2019, 9, 16252

Received 19th April 2019

Accepted 18th May 2019

DOI: 10.1039/c9ra02946j

rsc.li/rsc-advances

\section{Enhanced efficiency and high temperature stability of hybrid quantum dot light-emitting diodes using molybdenum oxide doped hole transport layer}

\author{
Jinyoung Yun, ${ }^{a}$ Jaeyun Kim, ${ }^{b}$ Byung Jun Jung, ${ }^{c}$ Gyutae Kim (D) *a \\ and Jeonghun Kwak iD *d
}

\begin{abstract}
High power efficiency (PE) and stability of quantum dot (QD) light-emitting diodes (QLEDs) are important factors for practical use in various displays. However, hybrid QLEDs consisting of an organic hole transport layer $(\mathrm{HTL})$ and an inorganic electron transport layer (ETL) sometimes have poor stability due to the low thermal stability of the organic HTL. To solve the problem, here, we report enhanced efficiency, lifetime, and temperature stability in inverted and hybrid structured QLEDs by adopting a $\mathrm{MoO}_{3}$-doped HTL. Also, to improve the electron-hole charge carrier balance, a thin insulating interlayer was used between QDs and the ETL. As a result, the QLED with the p-doped HTL exhibited the increased PE by $\sim 28 \%$ and longer lifetime compared to the pristine QLEDs. In addition, the QLED showed stable operation at the high temperature up to $400 \mathrm{~K}$, whereas the control device failed to operate at $375 \mathrm{~K}$. We systematically investigated the effect of the $\mathrm{MoO}_{3}$-doping on the performance and thermal stability of the QLEDs. We believe that QLEDs with the p-doped HTL can be used for further QLED researches to simultaneously improve the efficiency, lifetime, and high temperature stability, which are highly required for their use in automotive and outdoor displays.
\end{abstract}

\section{Introduction}

Colloidal nanocrystal quantum dots (QDs) exhibit several unique characteristics as an emitter, such as high photoluminescence (PL) quantum yields (QYs), size-controlled tunability of emission wavelengths, and narrow emission spectra based on a strong quantum confinement effect. ${ }^{1-6}$ Due to the outstanding optical properties, QDs attract attention for full colour display devices with a wide colour gamut (WCG) and high power efficiency. In particular, since the first QD-based light-emitting diodes (QLEDs) were introduced about two decades ago, ${ }^{7}$ their performance has been rapidly improved though multidisciplinary, extensive researches and

${ }^{a}$ School of Electrical Engineering, Korea University, Seoul 02841, South Korea. E-mail: gtkim@korea.ac.kr

${ }^{b}$ School of Electrical and Computer Engineering, University of Seoul, Seoul 02504, South Korea

'Department of Materials Science and Engineering, University of Seoul, Seoul 02504, South Korea

${ }^{d}$ Department of Electrical and Computer Engineering, Inter-University Semiconductor Research Center, Seoul National University, Seoul 08826, South Korea. E-mail: jkwak@snu.ac.kr

$\dagger$ Electronic supplementary information (ESI) available: UV-Vis absorption, PL, and EL spectra of CdZnSeS/ZnS QDs and QLEDs, $J-V$ characteristics of the HODs without and with the p-doped layer, $C-V$ and $R-V$ characteristics of the QLEDs, and AFM images of CBP and CBP/CBP: $\mathrm{MoO}_{3}$ films annealed at 300-350 K for $30 \mathrm{~min}$. See DOI: 10.1039/c9ra02946j developments on QDs, ligands, and device structures. In particular, the maximum efficiency of Cd-based QLEDs are comparable with that of phosphorescent organic light-emitting diodes (OLEDs), ${ }^{8,47}$ which made QLEDs be one of the most promising candidates for the next-generation display devices. However, the QLEDs still need to be ameliorated in terms of the efficiency, lifetime, and temperature stability to cope with the expansion of display industry toward non-consumer electronic devices, such as automotive displays and public displays.

A plenty of QLED researches have been conducted based on organic-inorganic hybrid device structures consisting of n-type metal oxides (e.g., $\mathrm{ZnO}, \mathrm{SnO}_{2}$ or $\mathrm{TiO}_{2}$ ) as an electron transport layer (ETL) and p-type small molecules or polymers as a hole transport layer (HTL). ${ }^{9-15}$ This structure has several advantages, such as energy level alignment for facilitated electron/hole charge carrier injection into QDs and easy processability for the multi-layered stacking. As a result, hybrid QLEDs exhibit much higher performance in terms of efficiency, turn-on voltage $\left(V_{\text {on }}\right)$, and lifetime, compared to the devices using allorganic $^{6,16,17}$ or all-inorganic ${ }^{18}$ transport layers.

On the other hand, the hybrid device structure has a few demerits to be solved. The most typical issue is electron-hole carrier imbalance during operation. Several phenomena, such as hole leakage current at the imperfect interface between QDs and the metal oxide ETL, ${ }^{19}$ negative charging of QDs by excessive electrons in the metal oxide $\operatorname{ETL}^{20}$ and the intrinsic difference of the charge carrier density and mobility between 
inorganic ETLs and organic HTLs, ${ }^{21}$ are related to the electronhole imbalance. Thus, reduced efficiency, poor operational stability, and severe efficiency roll-off can be observed mostly in hybrid QLEDs. ${ }^{22-25}$ Recently, a few strategies have been introduced to improve the charge balance. Among the methods, inserting a thin interlayer between the QD emission layer (EML) and the metal oxide ETL is a widely used and effective strategy to control excess electron injection into QDs. For instance, a thin layer of insulating polymers, such as polymethyl methacrylate (PMMA), ${ }^{26,27}$ polyethylenimine ethoxylated (PEIE), ${ }^{28,29}$ polyethylenimine (PEI), ${ }^{30}$ and poly(4-vinylpyridine) (PVPy) ${ }^{31}$ was adopted at the EML/ETL interface. These insulating polymers efficiently suppress excess electron injection into QDs, excitonpolaron quenching and hole leakage current at the interface, leading to the improvement of the external quantum efficiency (EQE), current efficiency (CE), and operational stability of the QLEDs. However, reduced electron current by the insulating layer inevitably brings about high driving voltages and reduced power efficiency (PE), ${ }^{26,27,31}$ which deteriorates the merits of using an interlayer in QLEDs.

In this letter, we introduce a novel hybrid QLED structure exhibiting good electron-hole balance, mainly originating from (i) enhanced hole current by adopting a molybdenum trioxide $\left(\mathrm{MoO}_{3}\right)$ doped HTL (p-doping) and (ii) suppressed excess electron injection and hole leakage current by using a PVPy interlayer. These devices thereby could show high PE, low driving voltages, and enhanced lifetime. In addition, the incorporation of the $\mathrm{MoO}_{3}$ doped HTL enabled stable operation of the QLEDS at a high temperature condition of $\sim 400 \mathrm{~K}$, which is a primary requisite factor for automotive and outdoor applications.

\section{Experimental method}

Pre-patterned indium-tin-oxide (ITO) glass substrates were cleaned in an ultrasonic bath with acetone and de-ionized water and then dried in an oven at $120^{\circ} \mathrm{C}$. Green-emitting core/shell QDs in a chemical composition of $\mathrm{CdZnSeS} / \mathrm{ZnS}$ were purchased from Invisible, Inc. The UV-Vis absorption and PL spectra are displayed in Fig. S1(a) in ESI. $\uparrow$ ZnO nanoparticles (NPs) were synthesized according to the previously reported synthetic procedures ${ }^{32}$ and dispersed in butanol. Poly(4vinylpyridine) (PVPy, weight average molecular weight $\sim 60$ 000) and $\mathrm{MoO}_{3}$ were purchased from Sigma-Aldrich, and $4,4^{\prime}$-bis( $N$-carbazolyl)-1,1'-biphenyl (CBP) was purchased from Lumtec. All the commercial materials were used as received. The dispersion solution of ZnO NPs with a concentration of $30 \mathrm{mg} \mathrm{mL}^{-1}$ was spin-coated at $2000 \mathrm{rpm}$ for $60 \mathrm{~s}$ as the ETL and thermally annealed on a hot plate at $90{ }^{\circ} \mathrm{C}$ for $30 \mathrm{~min}$. PVPy dissolved in methanol with a concentration of $0.5 \mathrm{mg} \mathrm{mL}^{-1}$ and QDs dispersed in hexane with a concentration of $10 \mathrm{mg} \mathrm{mL}$ were sequentially spin-coated at $4000 \mathrm{rpm}$ for $30 \mathrm{~s}$ on the top of the $\mathrm{ZnO}$ layer, and then dried at $75^{\circ} \mathrm{C}$ for $10 \mathrm{~min}$, respectively. After drying, CBP, $\mathrm{MoO}_{3}$, and $\mathrm{Al}$ as the HTL, HIL, and anode, respectively, were thermally evaporated under a high vacuum pressure of $<3 \times 10^{-7}$ torr. For the metal oxide-doped HTL, CBP and $\mathrm{MoO}_{3}$ were co-deposited in the same chamber. The QLED device structure and the energy band diagrams are depicted in Fig. 1(a) and (b), respectively.

The current density $(J)$-voltage $(V)$-luminance $(L)$ properties and the electroluminescence (EL) spectra were measured using a Keithley 2400 source measure unit and a Konica Minolta CS2000 spectroradiometer. The temperature dependent $J-V$ characteristics were obtained using a liquid nitrogen cooled cryostat system while the temperature was controlled using a proportional-integral-derivative (PID) temperature controller (LakeShore Model 335 Cryogenic Temperature Controller) which monitors the sample temperature using two silicon diode sensors. The absorption spectra were characterized using a UVVis spectrophotometer (Shimadzu UV3105PC). The surface morphology was obtained using an atomic force microscope (AFM, Park Systems NX10) in a non-contact mode. The operating temperature distribution and infrared (IR) images of the QLEDs were measured using an IR camera (FLIR A325sc). The capacitance $(C)-V$ and resistance $(R)-V$ curves were measured using an LCR meter (Hewlett Packard 4284A) with an oscillation amplitude of $25 \mathrm{mV}$ and a frequency of $10 \mathrm{kHz}$.

\section{Results and discussion}

Among several transition metal oxides (TMOs), $\mathrm{MoO}_{3}$ was chosen as the p-type dopant in this work by considering the thermal evaporation temperature and the energy levels of the host material (i.e., CBP) for higher doping efficiency. ${ }^{33,34}$ To investigate the effects of using a $\mathrm{MoO}_{3}$ doped HTL on the device performance, we fabricated green-emitting QLEDs with four different structures as follows:

Device A1: ITO/ZnO $(40 \mathrm{~nm}) / \mathrm{QD}(20 \mathrm{~nm}) / \mathrm{CBP}(50 \mathrm{~nm}) / \mathrm{MoO}_{3}$ $(10 \mathrm{~nm}) / \mathrm{Al}(80 \mathrm{~nm})$

Device A2: ITO/ZnO (40 nm)/QD (20 nm)/CBP (30 nm)/ CBP: $\mathrm{MoO}_{3}(20 \mathrm{~nm}, 10 \mathrm{vol} \%) / \mathrm{MoO}_{3}(10 \mathrm{~nm}) / \mathrm{Al}(80 \mathrm{~nm})$.

Device B1: ITO/ZnO (40 nm)/PVPy $(4 \mathrm{~nm}) / \mathrm{QD}(20 \mathrm{~nm}) / \mathrm{CBP}$ $(50 \mathrm{~nm}) / \mathrm{MoO}_{3}(10 \mathrm{~nm}) / \mathrm{Al}(80 \mathrm{~nm})$

Device B2: ITO/ZnO (40 nm)/PVPy (4 nm)/QD (20 nm)/CBP $(30 \mathrm{~nm}) / \mathrm{CBP}: \mathrm{MoO}_{3}(20 \mathrm{~nm}, 10 \mathrm{vol} \%) / \mathrm{MoO}_{3}(10 \mathrm{~nm}) / \mathrm{Al}(80 \mathrm{~nm})$.

Device A1 is a control device with a non-doped HTL (50 nm), whereas Device A2 has a HTL which consists of a p-doped HTL $(20 \mathrm{~nm})$ and a non-doped HTL (30 nm). In general, $\mathrm{MoO}_{3}$-doped organic thin films exhibit enhanced hole transporting properties by forming charge-transfer (CT) complexes, ${ }^{33}$ as reported in several optoelectronic devices, such as OLEDs and organic
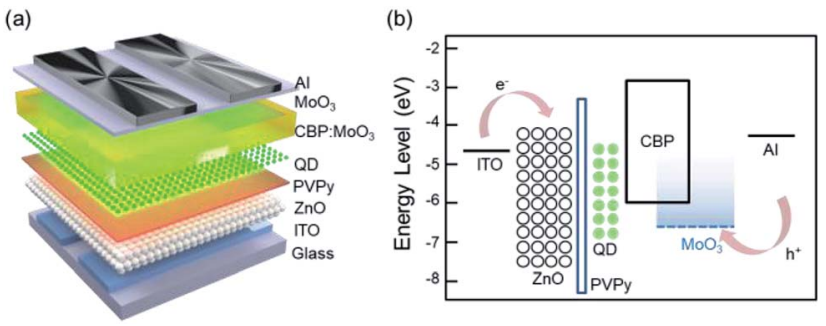

Fig. 1 (a) Inverted QLED device structure and (b) its energy band diagram. 

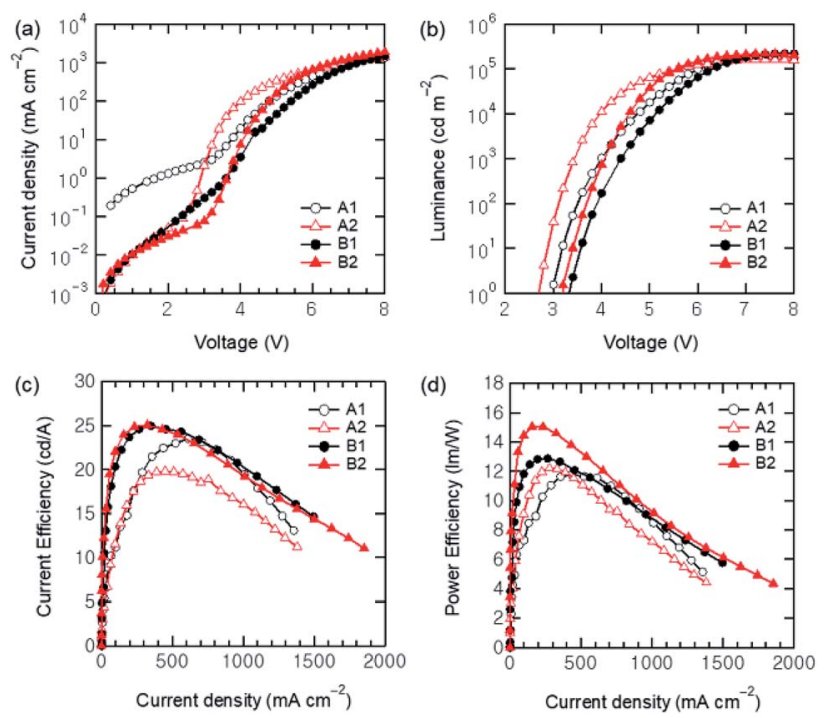

Fig. 2 (a) Current density-voltage, (b) luminance-voltage, (c) current efficiency-current density and (d) power efficiency-current density characteristics of the QLEDs.

photovoltaic cells. ${ }^{35-40}$ Similarly, it is supposed that applying a pdoped HTL to QLEDs will increase the hole current through the device. Especially, it is well known that hole injection from organic HTLs into Cd-based QDs is much harder than electron injection because of the large energy barrier between the highest occupied molecular orbital (HOMO) energy level of organic HTLs and the valence band maximum (VBM) energy levels of Cd-based QDs. Therefore, it can be thought that the pdoped HTL in QLEDs will be able to enhance overall device performance.

As shown in the $J-V$ and $L-V$ characteristics of the devices in Fig. 2, the current density of Device A2 is much higher than Device A1. The turn-on voltage (which corresponds to the voltage for $1 \mathrm{~cd} \mathrm{~m}^{-2}$ ) was also decreased with the p-doped HTL by $\sim 0.2 \mathrm{~V}$. As a result, the PE of Device A2 was higher than that of Device A1 at a low current density regime of approximately $<400 \mathrm{~mA} \mathrm{~cm}^{-2}$ where the electron charge carriers are abundant. At this regime, the $\mathrm{CE}$ of Devices A1 and A2 was almost identical. At a higher current density regime $\left(J>400 \mathrm{~mA} \mathrm{~cm}{ }^{-2}\right)$, however, the CE and PE of Device A2 with the $\mathrm{MoO}_{3}$-doped HTL became noticeably lower than those of Device A1 without a pdoped HTL. In this case, high $J$ is attributed to the increment of hole carriers, resulting in hole leakage current at the QD/ZnO interface and/or exciton quenching by excess hole carriers. We could thus know that the QLED performance as well as the charge balance cannot be improved for entire bias ranges by solely enhancing the hole transporting properties. The enhancement of hole transport properties of the $\mathrm{MoO}_{3}$-doped CBP film was confirmed with hole only devices (HODs) in the structure of ITO/ $\mathrm{MoO}_{3}(10 \mathrm{~nm}) / \mathrm{CBP}(30 \mathrm{~nm}) / \mathrm{CBP}: \mathrm{MoO}_{3}(20 \mathrm{~nm}$, $10 \mathrm{vol} \%)$ or $\mathrm{CBP}(20 \mathrm{~nm}) / \mathrm{MoO}_{3}(10 \mathrm{~nm}) / \mathrm{Al}(80 \mathrm{~nm})$. As plotted in Fig S2 in ESI, $\dagger$ the HOD with the p-doped HTL exhibited higher $J$ and the conductance than that without doping.

To improve the charge balance with utilizing the p-doped HTL, we inserted a thin interlayer of PVPy between QDs and ZnO NPs into Device A1 and A2, designated as Devices B1 (normal HTL) and B2 (p-doped HTL), respectively. As reported previously, this non-conjugated PVPy layer can not only lower excess electron injection but also block reduce hole leakage current. $^{31}$ The same results are shown in Fig. 2(a). When comparing Device A1 (without PVPy) and B1 (with PVPy), the efficiency of Device B1 at a low $J$ regime is much higher than that of Device A1. Meanwhile, the $J$ of both Devices B1 and B2 is lower than that of Devices A1 and A2, respectively, because of the insulating property of PVPy. Here, due to the p-doping, the $J$ of Device B2 is higher than that of Device B1. Device B2 also exhibits higher luminance at a same voltage and lower $V_{\text {on }}$ of $\sim 3.1 \mathrm{~V}$, compared to those of Device B1 with $V_{\text {on }}$ of $\sim 3.3 \mathrm{~V}$, as shown in Fig. 2(b). The QLEDs with a PVPy layer exhibit a bit higher maximum luminance $\left(L_{\max }\right)$ of over $210000 \mathrm{~cd} \mathrm{~m} \mathrm{~m}^{-2}$, compared with the devices without a PVPy layer. The maximum CE of Devices B1 and B2 is similar as approximately $25 \mathrm{~cd} \mathrm{~A}^{-1}$, which is higher than that of the devices without PVPy, as can be seen in Fig. 2(c), originating from the improved charge balance. Moreover, it is notable that the PE of Device B2 is superior to the other QLEDs over the entire current density regions, as shown in Fig. 2(d). The maximum PE of Device B2 was $15.03 \mathrm{~lm} \mathrm{~W}^{-1}$, while those of Devices A1, A2, and B1 were 11.76, 12.20, and $12.88 \mathrm{~lm} \mathrm{~W}^{-1}$, respectively. Device $\mathrm{B} 2$ also endured the maximum $J$ up to $\sim 1.9 \mathrm{~A} \mathrm{~cm}^{-2}$, which was the highest value among the devices. This result indicates that adopting both the $\mathrm{MoO}_{3}$-doped HTL and the PVPy interlayer is an effective method to improve the CE and PE simultaneously in hybrid QLEDs. In all the devices, the EL peak wavelength $\left(\lambda_{\max }\right)$ was observed identically at $523 \mathrm{~nm}$ with a full width at half maximum (FWHM) bandwidth of $22 \mathrm{~nm}$ (Fig S1 in ESI $\dagger$ ), meaning that $\mathrm{MoO}_{3}$-doping in the HTL have no influence on the optical properties of the QLEDs. The performance of the QLEDs is also summarized in Table 1.

We also investigated the $J-V$ characteristics of Devices B1 and $\mathrm{B} 2$ in the temperature range of 100-400 K, as shown in Fig. 3. At $100 \mathrm{~K}$, Device B1 shows a trap-limited space-charge-limitedcurrent (SCLC, $J \propto V^{2}$ ) shape at the bias conditions of $<6 \mathrm{~V}$.

Table 1 Device characteristics with four different structures

\begin{tabular}{llllllrr}
\hline Device & $V_{\text {on }}(\mathrm{V})$ & $\mathrm{EQE}(\%)$ & $\mathrm{CE}\left(\mathrm{cd} \mathrm{A}^{-1}\right)$ & $\mathrm{PE}\left(\mathrm{lm} \mathrm{W} \mathrm{m}^{-1}\right)$ & $L_{\max }\left(\mathrm{cd} \mathrm{m} \mathrm{m}^{-2}\right)$ & $\lambda_{\max }(\mathrm{nm})$ & $\mathrm{FWHM}(\mathrm{nm})$ \\
\hline A1 & 2.9 & 5.72 & 23.52 & 11.76 & 197000 & 523 & 22 \\
A2 & 2.7 & 4.90 & 19.82 & 12.20 & 164000 & 523 & 22 \\
B1 & 3.3 & 6.19 & 24.98 & 12.88 & 220000 & 523 & 22 \\
B2 & 3.1 & 6.24 & 25.07 & 15.03 & 216000 & 523 & 22
\end{tabular}



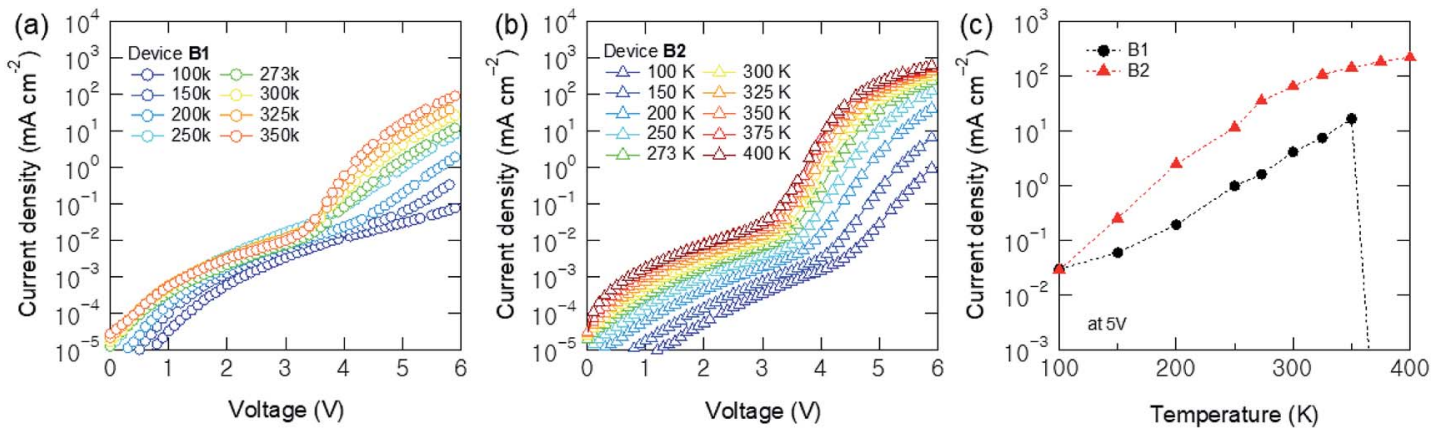

Fig. $3 \mathrm{~J}-V$ characteristics of the PVPy-inserted QLEDs with the (a) non-doped and (b) p-doped HTL, measured at 100-400 K. Device B1 failed to operate at $375 \mathrm{~K}$. (c) Comparison of temperature dependent $J$ values of two devices at $5 \mathrm{~V}$.
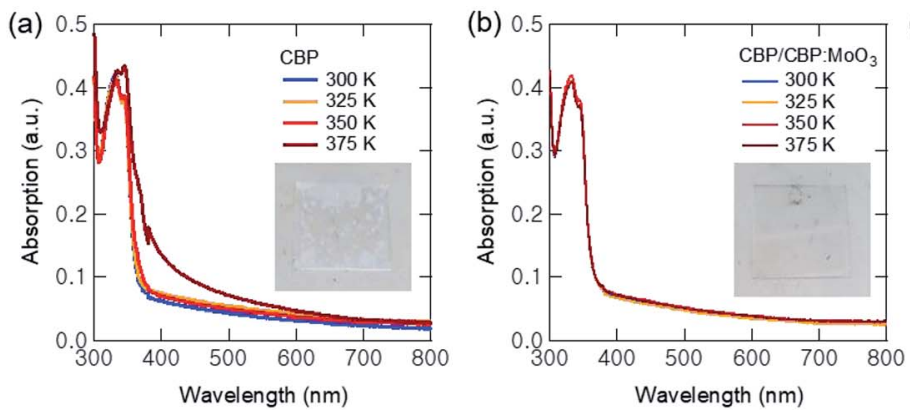

(c)

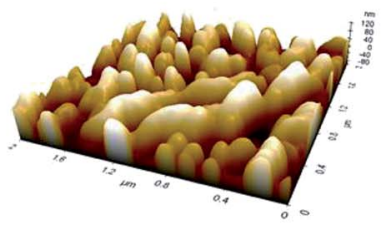

(d)

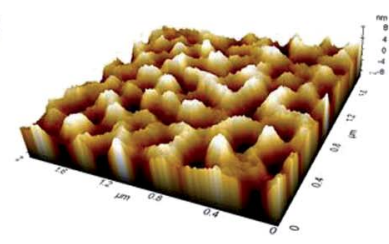

Fig. 4 UV-Vis absorption spectra of the (a) CBP $(50 \mathrm{~nm})$ and (b) CBP $(30 \mathrm{~nm}) / C B P: \mathrm{MoO}_{3}(20 \mathrm{~nm}, 10$ vol\%) films measured at various annealing temperatures of 300-375 K. Inset: photographs taken at the annealing temperature of $375 \mathrm{~K}$. The surface morphology ( $2 \mu \mathrm{m} \times 2 \mu \mathrm{m})$ of the (c) $\mathrm{CBP}(50 \mathrm{~nm})$ and (d) CBP $(30 \mathrm{~nm}) / \mathrm{CBP}: \mathrm{MoO}_{3}(20 \mathrm{~nm}, 10$ vol\%) films measured using an AFM at $375 \mathrm{~K}$.

Then, at $150 \mathrm{~K}$, a trap-filled limit (TFL, $\left.J \propto V^{\alpha}, \alpha^{>} 2\right)$ voltage $\left(V_{\text {TFL }}\right.$ ) can be observed as $\sim 5 \mathrm{~V}$ and decreased down to $\sim 3.5 \mathrm{~V}$ as the temperature increases gradually. Meanwhile, Device B2 with a $\mathrm{MoO}_{3}$-doped HTL shows a typical temperature dependent $J-V$ curves with the $V_{\mathrm{TFL}}$ ranging from $\sim 4.5 \mathrm{~V}$ to $\sim 3.2 \mathrm{~V}$, which were lower than those of Device B1. In other words, hole carrier concentration was increased by p-doping for all the measured temperature ranges. It is also supported by the $C-V$ and $R-V$ characteristics plotted in Fig. S3 in ESI. $\dagger$ The peak $C$ of Device B2 (3.25 nF) was much higher than that of Device B1 (1.85 nF). The $R$ at $V_{\text {on }}$ was $113 \mathrm{k} \Omega$ for Device B1, and it was decreased to 51 $\mathrm{k} \Omega$ for Device B2. These results indicate that partial $\mathrm{MoO}_{3}$ doping increases hole carrier concentration and thus increases the hole transport ability of the CBP layer.

It is also noteworthy that the operating temperature of the QLEDs were highly improved by adopting a $\mathrm{MoO}_{3}$-doped HTL. Device B2 with a doped HTL showed stable operation at the temperature of up to $400 \mathrm{~K}$, as shown in Fig. 3(b). Device B1 with the non-doped HTL showed overall normal operation until the temperature was increased to $350 \mathrm{~K}$ (Fig. 3(a)). However, severe degradation was observed at $375 \mathrm{~K}$ and the device was failed to operate. As mentioned above, stable operation under a high temperature condition is an essential factor for the practical use of QLEDs in automotive and outdoor displays. Fig. 3(c) compares the temperature dependent $J$ values of two devices at $5 \mathrm{~V}$.
To find out the reasons, we paid attention to the low glass transition temperature $\left(T_{\mathrm{g}}\right)$ of CBP, which is $62{ }^{\circ} \mathrm{C} .{ }^{43}$ Aggregation or crystallization can be occurred in organic thin films at high temperature above $T_{\mathrm{g}}$. We prepared two organic thin films with the same configuration of the QLEDs, which were CBP (50 nm) and CBP (30 nm)/CBP: $\mathrm{MoO}_{3}(20 \mathrm{~nm}, 10 \mathrm{vol} \%)$ films, on quartz substrates. Then, we annealed the films on a hot plate by changing the temperature from $300 \mathrm{~K}$ to $375 \mathrm{~K}$ for $30 \mathrm{~min}$, and compared their UV-Vis absorption spectra. As shown in the inset photograph and curves in Fig. 4(a), the CBP-only film started to crystallize from $375 \mathrm{~K}$, showing a broad absorption spectrum and white coloured aggregation
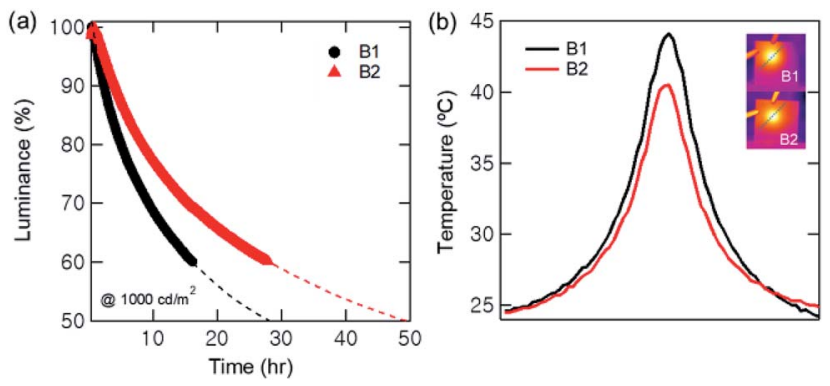

Fig. 5 (a) Operational lifetime of Devices B1 and B2. Dashed lines are the predicted lifetime using the stretched-exponential function. (b) Operating temperature profile of Devices B1 and B2 through the dotted lines in the thermal images using IR camera (inset). 
of the molecules. However, the p-doped film shows no spectral and visual change while the annealing temperature changed from $300 \mathrm{~K}$ to $375 \mathrm{~K}$, as shown in Fig. 4(b). Therefore, we can attribute the failure of Device B1 to the low $T_{\mathrm{g}}$ of the CBP HTL, and the improved temperature stability of Device B2 to the enhanced $T_{\mathrm{g}}$ of the p-doped CBP HTL, respectively. The surface morphology of the CBP and CBP/ CBP: $\mathrm{MoO}_{3}$ films annealed at $375 \mathrm{~K}$ is compared in Fig. 4(c) and (d). The root-mean-square (RMS) roughness $\left(R_{\mathrm{q}}\right)$ of the CBP-only film was as high as $\sim 49 \mathrm{~nm}$ due to the severe aggregation/crystallization of CBP, whereas that of the $\mathrm{MoO}_{3}$ doped CBP film was $\sim 2 \mathrm{~nm}$, at $375 \mathrm{~K}$. At 300-350 K, both films exhibited the $R_{\mathrm{q}}$ of about 1-2 nm with little difference (see Fig. S4 in $\mathrm{ESI}_{\dagger}^{\dagger}$ ).

We also measured the operational lifetime of the QLEDs at the initial luminance of $1000 \mathrm{~cd} \mathrm{~m}^{-2}$, as shown in Fig. 5(a). The half lifetime was predicted by data fitting to the stretchedexponential function $L(t)$ with the shape parameter $\beta=1$,

$$
L(t)=L_{0} \exp \left[-(t / \tau)^{\beta}\right]
$$

where $L_{0}$ is initial luminance, $t$ is the operating time and $\tau$ corresponds to the decay time. ${ }^{\mathbf{4 1 , 4 2}}$ The half lifetime of Device B2 is expected to be almost 2 times longer than that of Device B1. The lifetime enhancement of Device B2 originated from the improved efficiency and lower driving voltages. Also, higher $T_{\mathrm{g}}$ generally improves the operational stability of the devices. ${ }^{\mathbf{4 4 - 4 6}}$ Thus we observed the operating temperature distribution of the QLEDs using an IR camera. The thermal images in the inset of Fig. 5(b) were taken under the bias condition of $J=390 \mathrm{~mA}$ $\mathrm{cm}^{-2}$, which corresponds to the luminance of $\sim 10^{5} \mathrm{~cd} \mathrm{~m}^{-2}$ for both devices. The operating temperature of Device B1 is slightly higher (by $\sim 4{ }^{\circ} \mathrm{C}$ ) than that of Device B2. The difference is not significant, but this operating temperature can influence on the long-term lifetime of the QLEDs. Therefore, we could suggest that adopting a p-doped HTL into the QLEDs is a descent method to improve the efficiency, lifetime, and thermal stability, at once.

\section{Conclusions}

We demonstrated enhanced performance and stability in inverted QLEDs using a $\mathrm{MoO}_{3}$-doped HTL and a PVPy interlayer. With the device structure, we could achieve increased hole injection, reduced hole leakage current, longer lifetime, and high temperature operation. The reasons for the improvement were enhanced thermal stability of the doped HTL and the improved electron-hole balance. We believe that the simple codeposition method can be applicable for further QLED researches and the development of automotive and outdoor display devices, with improve the efficiency, lifetime, and temperature stability.

\section{Conflicts of interest}

There are no conflicts to declare.

\section{Acknowledgements}

This work was supported by the Industrial Technology Innovation Program (No. 10052853) funded by the Ministry of Trade, Industry \& Energy (MOTIE, Korea).

\section{References}

1 Y. Shirasaki, G. J. Supran, M. G. Bawendi and V. Bulović, Nat. Photonics, 2013, 7, 13-23.

2 V. Wood and V. Bulović, Nano Rev., 2010, 1, 1-7.

3 Y. Yang, Y. Zheng, W. Cao, A. Titov, J. Hyvonen, J. R. Manders, J. Xue, P. H. Holloway and L. Qian, Nat. Photonics, 2015, 9, 259-266.

4 J. Kwak, J. Lim, M. Park, S. Lee, K. Char and C. Lee, Nano Lett., 2015, 15, 3793-3799.

5 G. J. Supran, Y. Shirasaki, K. W. Song, J. Caruge, P. T. Kazlas, S. Coe-Sullivan, T. L. Andrew, M. G. Bawendi and V. Bulović, MRS Bull., 2013, 38, 703-711.

6 S. Coe, W. Woo, M. Bawendi and V. Bulović, Nature, 2002, 420, 800-803.

7 V. L. Colvin, M. C. Schlamp and A. P. Alivisatos, Nature, 1994, 370, 354-357.

8 H. J. Jang, J. Y. Lee, J. Kwak, D. Lee, J. Park, B. Lee and Y. Y. Noh, J. Inf. Disp., 2019, 20, 1-8.

9 T.-H. Kim, K.-S. Cho, E. K. Lee, S. J. Lee, J. Chae, J. W. Kim, D. H. Kim, J.-Y. Kwon, G. Amaratunga, S. Y. Lee, B. L. Choi, Y. Kuk, J. M. Kim and K. Kim, Nat. Photonics, 2011, 5, 176182.

10 L. Qian, Y. Zheng, J. Xue and P. H. Holloway, Nat. Photonics, 2011, 5, 543-548.

11 J. Kwak, W. K. Bae, D. Lee, I. Park, J. Lim, M. Park, H. Cho, H. Woo, D. Y. Yoon, K. Char, S. Lee and C. Lee, Nano Lett., 2012, 12, 2362-2366.

12 Q. Sun, Y. A. Wang, L. S. Li, D. Wang, T. Zhu, J. Xu, C. Yang and Y. Li, Nat. Photonics, 2007, 1, 717-722.

13 W. Ji, Y. Lv, P. Jing, H. Zhang, J. Wang, H. Zhang and J. Zhao, ACS Appl. Mater. Interfaces, 2015, 7, 15955-15960.

14 H. Peng, W. Wang and S. Chen, IEEE Electron Device Lett., 2015, 36, 369-371.

15 C. Jiang, H. Liu, B. Liu, Z. Zhong, J. Zou, J. Wang, L. Wang, J. Peng and Y. Cao, Org. Electron., 2016, 31, 82-89.

16 P. O. Anikeeva, J. E. Halpert, M. G. Bawendi and V. Bulović, Nano Lett., 2009, 9, 2532-2536.

17 J. Zhao, J. A. Bardecker, A. M. Munro, M. S. Liu, Y. Niu, I. Ding, J. Luo, B. Chen, A. K.-Y. Jen and D. S. Ginger, Nano Lett., 2006, 6, 463-467.

18 J. M. Caruge, J. E. Halpert, V. Wood, V. Bulović and M. G. Bawendi, Nat. Photonics, 2008, 2, 247-250.

19 S. Coe-Sullivan, W. Woo, J. S. Steckel, M. Bawendi and V. Bulovic, Org. Electron., 2003, 4, 123-130.

20 W. K. Bae, Y. Park, J. Lim, D. Lee, L. A. Padilha, H. McDaniel, I. Robel, C. Lee, J. M. Pietryga and V. I. Klimov, Nat. Commun., 2013, 4, 2661.

21 J. H. Chang, P. Park, H. Jung, B. G. Jeong, D. Hahm, G. Nagamine, J. Ko, J. Cho, L. A. Padilha, D. C. Lee, C. Lee, K. Char and W. K. Bae, ACS Nano, 2018, 12, 10231-10239. 
22 W. Xu, W. Ji, P. Jing, X. Yuan, Y. A. Wang, W. Xiang and J. Zhao, Opt. Lett., 2014, 39, 426-429.

23 Y. Ran, J. Hoon, K. Shin, Y. Soo, Y. Seok, S. Young, W. Kook and Y. Joon, Org. Electron., 2015, 19, 131-139.

24 T. Ding, X. Yang, L. Ke, Y. Liu, W. Tan and N. Wang, Org. Electron., 2016, 32, 89-93.

25 Y. R. Cho, P. G. Kang, D. H. Shin, J. H. Kim, M. J. Maeng, J. Sakong, J. A. Hong, Y. Park and M. C. Suh, Appl. Phys. Express, 2016, 9, 012103.

26 X. Dai, Z. Zhang, Y. Jin, Y. Niu, H. Cao, X. Liang, L. Chen, J. Wang and X. Peng, Nature, 2014, 515, 96-99.

27 Q. Lin, L. Wang, Z. Li, H. Shen, L. Guo, Y. Kuang, H. Wang and L. S. Li, ACS Photonics, 2018, 5, 939-946.

28 H. H. Kim, S. Park, Y. Yi, D. I. Son, C. Park, D. K. Hwang and W. K. Choi, Sci. Rep., 2015, 5, 8968.

29 D. Kim, Y. Fu, S. Kim, W. Lee, K. Lee, H. K. Chung, H. Lee, H. Yang and H. Chae, ACS Nano, 2017, 11, 1982-1990.

30 K. Ding, H. Chen, L. Fan, B. Wang, Z. Huang, S. Zhuang, B. Hu and L. Wang, ACS Appl. Mater. Interfaces, 2017, 9, 20231-20238.

31 J. Yun, J. Kim, H. K. Jang, K. J. Lee, J. H. Seo, B. J. Jung, G. Kim and J. Kwak, Org. Electron., 2017, 50, 82-86.

32 J. Kwak, J. Lim, M. Park, S. Lee, K. Char and C. Lee, Nano Lett., 2015, 15, 3793-3799.

33 S. Hamwi, J. Meyer, T. Winkler, T. Riedl and W. Kowalsky, Appl. Phys. Lett., 2009, 94, 253307.

34 M. Kröger, S. Hamwi, J. Meyer, T. Riedl, W. Kowalsky and A. Kahn, Org. Electron., 2009, 10, 932-938.

35 C. Chang, M. Hsieh, J. Chen, S. Hwang and C. H. Chen, Appl. Phys. Lett., 2006, 89, 253504.
36 D. Leem, H. Park, J. Kang, J. Lee, J. W. Kim and J. Kim, Appl. Phys. Lett., 2007, 91, 011113.

37 C. C. Chang, M. T. Hsieh, J. F. Chen, S. W. Hwang and C. H. Chen, Appl. Phys. Lett., 2006, 89, 253504.

38 H. Ikeda, J. Sakata, M. Hayakawa, T. Aoyama, T. Kawakami, K. Kamata, Y. Iwaki, S. Seo, Y. Noda, R. Nomura and S. Yamazaki, SID Int. - Symp. Dig. Tech. Pap., 2006, 37, 923.

39 X. L. Zhu, J. X. Sun, H. J. Peng, Z. G. Meng, M. Wong and H. S. Kwok, Appl. Phys. Lett., 2005, 87, 153508.

40 Y. Shinmura, M. Kubo, T. Kaji and M. Hiramoto, Jpn. J. Appl. Phys., 2013, 52, 04CR12.

41 G. Williams and D. C. Watts, Trans. Faraday Soc., 1970, 66, 80-85.

42 C. Féry, B. Racine, D. Vaufrey, H. Doyeux and S. Cinà, Appl. Phys. Lett., 2005, 87, 213502.

43 M. H. Tsai, Y. H. Hong, C. H. Chang, H. C. Su, C. C. Wu, A. Matoliukstyte, J. Simokaitiene, S. Grigalevicius, J. V. Grazulevicius and C. P. Hsu, Adv. Mater., 2007, 19, 862-866.

44 J. Kwak, Y. Lyu, S. Noh, H. Lee, M. Park, B. Choi, K. Char and C. Lee, Thin Solid Films, 2012, 520, 7157-7163.

45 J. A. McEwan, A. J. Clulow, A. Nelson, N. R. Yepuri, P. L. Burn and I. R. Gentle, ACS Appl. Mater. Interfaces, 2017, 9, 1415314161.

46 R. Braveenth, H. W. Bae, I. J. Ko, W. Qiong, Q. P. B. Nguyen, P. G. S. Jayashantha, J. H. Kwon and K. Y. Chai, Org. Electron., 2017, 51, 463-470.

47 H. Shen, Q. Gao, Y. Zhang, Y. Lin, Q. Lin, Z. Li, L. Chen, Z. Zeng, X. Li, Y. Jia, S. Wang, Z. Du, L. S. Li and Z. Zhang, Nat. Photonics, 2019, 13, 192-197. 